\title{
The application of $\gamma$-matrices to Taylor series
}

\author{
By P. Vermes \\ (Received 26th November, $1945 . \quad$ Read 8th December, 1945. \\ Revised paper received 13th July, 1946.)
}

1. Introduction. In a recent paper ${ }^{1}$ some general properties of $\gamma$-matrices were proved and Dienes' theorem on regular $\gamma$-matrices ${ }^{2}$ extended to semiregular $\gamma$-matrices and the binomial series. ${ }^{3}$ In section 2 of this paper the previous results will be extended to certain classes of Taylor series. Section 3 gives some new results on Borel's exponential summation, and section 4 introduces matrices efficient for Taylor series on the circle of convergence and others efficient for Dirichlet series on the line of convergence. A knowledge of the definitions and results of the paper mentioned above is assumed.

2. On the $\gamma$-sum of the Taylor series.

[2.I] If the semiregular $\gamma$-matrix $G$ sums the Taylor series $\Sigma a_{k} z^{k}$ of the function $f(z)$ at $z=z_{0}$ to the value $S$, then it also sums the Taylor series of the function $F(z) \equiv z^{p} f(z)(p=1,2, \ldots)$ at $z=z_{0}$ to the sum $z_{0}{ }^{p} S$.

Proof: By hypothesis $\lim _{n \rightarrow \infty} \sum_{k=0}^{\infty} g_{n, k} a_{k} z_{i}^{k}=S$, and, $G$ being semiregular,

$$
\lim _{n \rightarrow \infty} \sum_{k=0}^{\infty} g_{n, k+p} a_{k} z_{0}^{k}=S,
$$

which multiplied by $z_{0}^{p}$ can be rewritten

$$
\lim _{n \rightarrow \infty} \sum_{k=p}^{\infty} g_{n, k} a_{k-p} z_{0}^{k}=z_{0}^{p} S
$$

But the Taylor series of $F(z)$ is $\sum_{k=0}^{\infty} a_{k} z^{k+p}=\sum_{k=p}^{\infty} a_{k-p} z^{k}$, and so (2.1) proves the theorem.

Corollary. Under the conditions of the theorem, if $P(z)$ is a polynomial and $F(z) \equiv P(z) f(z)$, then $G$ sums the Tuylor series of $F(z)$ at $z_{0}$ to $P\left(z_{0}\right) S$.

1 P. Vermes, "On $\gamma$-matrices and their application to the binomial series," these Proceedings 8 (1947), 1-13. This paper will be referred to as $\gamma-M$.

2 P. Dienes, The Taylor Series (Oxford), 1931, 418. This book will be referred to as $T . S$.

$3 \cdot M$, section 5 . 
[2.II] If a semiregular $\gamma$-matrix $G$ sums the Taylor series of a meromorphic function $f(z)$ at a regular point $z=z_{0}$, then the sum is the "right" value $f\left(z_{0}\right)$.

Proof: Let $f(z)=\Sigma a_{k} z^{k}$ for $|z|<R$. By hypothesis if $\rho>\left|z_{0}\right|$, the only singularities of $f(z)$ in the circle $|z| \leqq \rho$ are poles $a_{i}$ of order $m_{i}$, so that if $F(z) \equiv P(z) f(z)$, where $P(z) \equiv \Pi\left(z-a_{i}\right)^{m_{i}}$ (the product being taken over all poles in the circle $|z| \leqq \rho), F(z)$ is analytic in and on this circle. Hence the Taylor series of $F(z)$ is convergent at $z_{0}$, and its $G$-sum therefore exists and is $P\left(z_{0}\right) f\left(z_{0}\right)$. Applying the Corollary of [2.I] we have $P\left(z_{0}\right) f\left(z_{0}\right)=P\left(z_{0}\right) S$, whence $S=f\left(z_{0}\right)$.

Corollary. The theorem readily extends to general Taylor series for values of $z_{0}$ in the circle of meromorphy.

[2.III] If the semiregular $\gamma$-matrix $G$ sums the series $\Sigma a_{k} z^{k}$ in the domain $D$ to $s(z)$, then the $\gamma$-matrix $H \equiv \sum_{i=0}^{\infty} \lambda_{i} G^{(i)} / \sum_{i=0}^{\infty} \lambda_{i}$ sums the series to the same value, provided that condition (b) of theorem [1.III] of $\gamma-M i_{s}$ satisfied by the $\lambda_{i}$, and that

(i) $\left|g_{n, k}\right| \geqq\left|g_{n, k+1}\right|$ for every $n$ and $k$,

(ii) $\left|\sigma_{i n}^{(i)}(z)\right| \equiv\left|\sum_{k=0}^{\infty} g_{n, k+i} a_{k} z^{k}\right| \leqq N(z)$ for every $i, n$, and a fixed $z$ in $D$. Moreover $H$ is semiregular with respect to this series. ${ }^{1}$

Note: It will be seen in [3.I] that (i) and under certain conditions (ii) hold for the Borel-matrix.

Proof: Since $g_{n, k}^{(i)}=g_{n, k+i}$ we see by [1.I] of $\gamma$-M that all conditions of [1.III] of $\gamma-M$ are satisfied. Hence $H$ exists and is a $\gamma$-matrix.

Since by hypothesis, for a fixed $n$, the series $\sigma_{n}^{0}(z) \equiv \Sigma g_{n, k} a_{k} z^{k}$ converges for every $z$ in $D$, it converges absolutely in $D$, i.e.

Also $\quad \sum_{k=0}^{\infty} \sum_{i=0}^{p}\left|\lambda_{i} g_{n, k}^{(i)} a z_{k} z^{k}\right|=\sum_{i=0}^{p}\left|\lambda_{i}\right| \sum_{k=0}^{\infty}\left|g_{n, k}^{(i)} a_{k} z^{k}\right|$

$$
\Sigma\left|g_{n, k} a_{k} z^{k}\right|=S_{n}(|z|) \text { is finite in } D \text {. }
$$

$$
\leqq \sum_{i=0}^{p}\left|\lambda_{i}\right| \sum_{k=0}^{\infty}\left|g_{n, k} a_{k} z^{k}\right| \leqq S_{n}(|z|) \sum_{i=0}^{\infty}\left|\lambda_{i}\right|=S_{n}(|z|) L .
$$

Since the last member is independent of $p$, the double series

$$
\rho_{n}(z) \equiv \sum_{k=0}^{\infty} \sum_{i=0}^{\infty} \lambda g_{n, k}^{(i)} a_{k} z^{k}
$$

1 Here $\left(^{(i)}\right.$ denotes the $i$-th diminutirc of $G$, e.g. (3.1) of $\gamma \cdot M$. 
converges absolutely for every $z$ in $D$, and thus we can reverse the order of summation, i.e.

$$
\rho_{n}(z)=\sum_{i=0}^{x} \lambda_{i} \sum_{k=0}^{\infty} g_{n, k}^{(i)} a_{k} z^{k} \equiv \sum_{i=0}^{\infty} \lambda_{i} \sigma_{n}^{(i)}(z) .
$$

Comparing the series on the right-hand side of (2.3) with the series $\Sigma\left|\lambda_{i}\right| N=N L$, we see that it converges uniformly for every $n$, so that

(2.4) $\lim _{n \rightarrow \infty} \rho_{n}(z)=\sum_{i=0}^{\infty} \lambda_{i} \lim _{n \rightarrow \infty} \sigma_{n}^{(i)}(z)=\sum_{i=0}^{\infty} \lambda_{i} s(z)=l s(z)$,

and it follows by (2.2) and (2.4) that

(2.5) $\lim _{n \rightarrow \infty} \sum_{k=0}^{\infty} h_{n, k} a_{k} z^{k}=\lim _{n \rightarrow \infty} \sum_{k=0}^{\infty}\left\{\begin{array}{ll}1 & \left.\sum_{i=0}^{\infty} \lambda_{i} g_{n, k}^{(i)}\right\}\end{array}\right\} a_{k} z^{k}=s(z)$.

i.e. $H$ sums ithe series io $s(z)$.

Also $H^{(1)} \equiv \Sigma \lambda_{i} G^{(i+1)} / \Sigma \lambda_{i}$ satisfies the conditions of this theorem. Hence (2.5) applies to $H^{(1)}$, showing that $H$ is semiregular with respect to $\Sigma a_{k} z^{k}$. This concludes the proof.

Corollary. If $\Sigma \lambda_{i}=0$, the matrix $\Sigma \lambda_{i} G^{(i)}$ (which is not a $\gamma$-matrix) sums the series to zero.

This follows from (2.4).

[2.IV] We suppose that $f(z) \equiv \Sigma a_{k} z^{k}$ in a circle $\Gamma$ round the origin, that the semiregular $\gamma$-matrix $G$ sums the series to $s(z)$ in the domain $D$, and that conditions (i) and (ii) of [z.III] are satisfied. If the function $F(z)$ is regular in a circle $C$ with centre at the origin, then $G$ sums the Taylor series of $F(z) f(z)$ about the origin to the sum $F(z) s(z)$ in the domain $C D$.

Proof: By hypothesis $F(z) \equiv \Sigma b_{i} z^{i}$ in $C$, whence

$$
F(z) f(z) \equiv \sum_{k=0}^{\infty} z^{k} \sum_{i=0}^{k} a_{k-i} b_{i} \text { in } C \Gamma
$$

and if we write $a_{i}=0$ for $\quad i=-1,-2,-3, \ldots$,

$$
F(z) f(z) \equiv \sum_{k=0}^{\infty} z^{k} \sum_{i=0}^{\infty} a_{k-i} b_{i} \quad \text { in } C \Gamma .
$$

By hypothesis

$$
\lim _{n \rightarrow \infty} \sum_{k=0}^{\infty} g_{n, k} a_{k} z^{k}=s(z) \quad \text { in } D \text {, and hence in } C D .
$$

Since the series $\Sigma b_{i} z^{i}$ converges absolutely in $C D$, we can apply [2.III] or its corollary with $\lambda_{i} \equiv b_{i} z^{i}$ and $l \equiv F(z)$ to $(2.7)$; and we have as in (2.2) and (2.4)

$$
\lim _{n \rightarrow \infty} \rho_{n}(z) \equiv \lim _{n \rightarrow \infty} \sum_{k=0}^{\infty} \sum_{i=0}^{\infty} b_{i} z^{i} g_{n, k+i} a_{k} z^{k}=F(z) s(z) \quad \text { in } C D .
$$


Writing $k-i$ for $k$, we have

$$
\lim _{n \rightarrow \infty} \sum_{k=i}^{\infty} g_{n, k^{2}} \sum_{i=0}^{\infty} b_{i} a_{k-i}=F(z) s(z) \quad \text { in } C D,
$$

and again putting $a_{i}=0$ for $i=-1,-2, \ldots$, we have

$$
\lim _{n \rightarrow \infty} \sum_{k=0}^{\infty} g_{n, k} z^{k} \sum_{i=0}^{\infty} a_{k-i} b_{i}=F(z) s(z) \quad \text { in } C D .
$$

The left-hand side is the $G$-sum of the series (2.6). Thus (2.8) proves the theorem.

3. Borel's exponential summation.

This is a summation method by the $\gamma$-matrix

$g_{n, k} \equiv \frac{1}{k !} \int_{0}^{n} e^{-t t^{k}} d t=1-e^{-n}\left(1+n+\frac{n^{2}}{2 !}+\ldots \frac{n^{k}}{k !}\right), k, n=0,1,2, \ldots$

The following well-known properties ${ }^{1}$ will be used in this section:

$$
\begin{aligned}
& g_{n, k} \geqq g_{n, k}+1 \geqq 0 \text { for every } n \text { and } k, \\
& g_{n, k} \rightarrow 0 \text { as } k \rightarrow \infty \text { for every fixed } n .
\end{aligned}
$$

When $G$ sums the series $\Sigma c_{k}$, the order of summation and integration. can be interchanged, ${ }^{2}$ i.e.

$$
\begin{aligned}
& \text { (B) sum of } \sum_{k=0}^{\infty} c_{k}=\lim _{n \rightarrow \infty} \sum_{k=0}^{\infty} \frac{c_{k}}{k !} \int_{0}^{n} e^{-t} t^{k} d t \\
& =\lim _{n \rightarrow \infty} \int_{0}^{n} e^{-t}\left(\sum_{k=0}^{\infty} \frac{c_{k} t^{k}}{k !}\right) d t=\int_{0}^{\infty} e^{-t}\left(\sum_{k=0}^{\infty} \frac{c_{k} t^{k}}{k !}\right) d t \text {. }
\end{aligned}
$$

$G$ is semiregular, and Hardy gave an example of a series summable by this method but with respect to which the summation is not regular. $^{3}$

Using the notation ${ }^{4} u_{0}(t) \equiv \sum_{k=0}^{\infty} \frac{c_{k} t^{k}}{k !}$,

we see from (3.2) that when $G$ sums the series $\Sigma c_{k}, u_{0}(t)$ is an integral function of $t$, and so $u_{0}(t)$ can be integrated repeatedly, giving

$$
u_{j}(t) \equiv \sum_{k=0}^{\infty} \frac{c_{k} t^{k+j}}{(k+j) !}
$$$$
j=0,1,2, \ldots .
$$

where $u_{j}(t)$ is an integral function of $t$. We also see that

$$
u_{j+1}(t)=\int_{0}^{t} u_{j}(t) d t, \quad j=0,1,2, \ldots
$$

I T.S. 401.

T.S. 401 .

3.S. 419-420.

4 T.S. 403-404. 
Since $G$ is semiregular, if $n \rightarrow \infty$

$$
\sigma_{n}^{0} \equiv \sum_{k=0}^{\infty} g_{n, k} c_{k} \rightarrow \delta \quad \quad \text { implies } \quad \sigma_{n}^{j} \equiv \sum_{k=0}^{\infty} g_{n, k+j} c_{k} \rightarrow s .
$$

Finally, we know that $\Sigma a_{k} z^{k}$ is summable by $G$, if $z$ is an inner point of the "polygon of summability," "to the "right" sum. $G$ is inefficient outside the polygon.

[3.I] If $\Sigma c_{k}$ is summable $(B)$, then $\left|\sigma_{n}^{j}\right|$ is bounded for every $n$ and $j$ whenever $\left|u_{0}(t)\right|$ is bounded for $0 \leqq t \leqq \infty$.

Proof: By (3.1), (3.2), (3.3), (3.4) and (3.5)

$$
\sigma_{n}^{j}=\int_{0}^{n} e^{-t} u_{j}(t) d t=\int_{0}^{n} e^{-t} u_{j-1}(t) d t+\left[e^{-t} u_{j}(t)\right]_{n}^{0} .
$$

(3.6) From (3.4) $u_{j}(0)=0$ for $j=1,2,3, \ldots$, and so by repeated integration by parts

$$
\sigma_{u}^{j}=\sigma_{n}^{0}-e^{-n}\left[u_{j}(n)+u_{j-1}(n)+\ldots+u_{1}(n)\right] .
$$

By (3.5) $\left|\sigma_{n}^{0}\right| \leqq K_{0}$ for every $n$. Also applying Taylor's theorem to $u_{j}(n)$ and considering (3.4) and (3.6), we have $u_{j}(n)=u_{0}\left(\Omega_{j}\right) n^{j} / j !$ where $0 \leqq \Omega_{j} \leqq n$. Hence

$$
\left|\sigma_{n}^{j}\right| \leqq K_{0}+e^{-n}\left[n\left|u_{0}\left(\Omega_{1}\right)\right|+\frac{n^{2}}{2 !}\left|u_{0}\left(\Omega_{2}\right)\right|+\ldots+\frac{n^{j}}{j !}\left|u_{0}\left(\Omega_{j}\right)\right|\right] \text {, }
$$

and, since by hypothesis $\left|u_{0}(t)\right| \leqq K$ for $0 \leqq t \leqq \infty$,

$$
\left|\sigma_{n}^{j}\right| \leqq K_{0}+e^{-n}\left[n+\frac{n^{2}}{2 !}+\ldots+\frac{n^{j}}{j !}\right] K \leqq K_{0}+K,
$$

which proves the theorem.

Examples. The divergent series $\Sigma(-2)^{T /}$ is summable $(B)$, and $u_{0}(t)=e^{-2 t}$ is bounded in $(0, \infty)$. The convergent series $\Sigma\left(\frac{1}{2}\right)^{k}$ is summable $(B)$, and $u_{0}(t)=e^{j t}$ is not bounded. But $u_{j}(n)$ and $\sigma_{n}^{j}$ are positive; hence by (3.7) $\sigma_{n}^{j}$ is bounded, so that the condition of this theorem is not necessary.

[3.II] Borel's $\gamma$-matrix is regular with respect to all Taylor series in the polygon of summability.

Proof: When $z_{0}=0$ the proof is trivial. If $z_{0} \neq 0$ is in the polygon of summability, then

1 T.S. 305. 
(3.8) $\lim _{n \rightarrow \infty} \sum_{k=0}^{\infty} g_{n, k} a_{k} z_{0}^{z}=a_{0}+\sum_{k=1}^{\infty} g_{n, k} a_{k} z_{0}^{k} \quad$ exists and is equal to $f\left(z_{0}\right)$. The function $F(z)=\left\{f(z)-a_{0}\right\} / z$ has singularities at the same finite points as $f(z)$ and at no other points. Hence it has the same polygon of summability. Thus $G$ sums the series for $F(z), \quad a_{1}+a_{2} z+a_{3} z^{2}+\ldots$, at $z_{0}$, i.e.

whence

$$
\lim _{n \rightarrow \infty} \sum_{k=1}^{\infty} g_{n, k-1} a_{k} z_{0}^{k-1}=F\left(z_{0}\right)=\frac{1}{z_{0}}\left\{f\left(z_{0}\right)-a_{0}\right\},
$$

$$
\lim _{n \rightarrow \infty} \sum_{k=1}^{\infty} g_{n, k-1} a_{k} z_{0}^{k}=f\left(z_{0}\right)-a_{0} .
$$

Comparing (3.8) and (3.9) we have for $z_{0}$ in the polygon of summability

$$
\lim _{n \rightarrow \infty} \sum_{k=1}^{\infty} g_{n, k} a_{k} z_{0}^{k} \rightrightarrows \lim _{n \rightarrow \infty} \sum_{k=1}^{\infty} g_{n, k-1} a_{k} z_{0}^{k}
$$

which proves the theorem, since the semiregularity of $G$ would be represented by (3.10) with the arrows reversed.

4. $\gamma$-matrices efficient on the boundaries of convergence-domains.

Given a sequencc $\rho_{0}, \rho_{1}, \rho_{2}, \ldots$ satisfying the conditions

$$
0<\rho_{n}<1 \text { for every } n,
$$

we construct the matrix $R$ :

$$
\begin{aligned}
& \rho_{n} \rightarrow 1 \text { as } n \rightarrow \infty, \\
r_{n, k}= & \rho_{n}{ }^{k+1} \quad(k, n=0,1,2, \ldots) .
\end{aligned}
$$

Then we have:

[4.I] $R$ is a regular $\gamma$-matrix, which sums every Taylor series at those points $z_{0}$ of its circle of convergence for which the function represented by the series tends to a limiting value when $z \rightarrow z_{0}$ along the radius.

Proof: By definition

$$
\begin{aligned}
& \sum_{k=0}^{\infty}\left|r_{n, k}-r_{n, k+1}\right|=\sum_{k=0}^{\infty}\left(r_{n, k}-r_{n, k+1}\right)=\rho_{n}<1 \text { for every } n \text {, and } \\
& \lim _{n \rightarrow \infty} r_{n, k}=1 \text { for every fixed } k \text {. Thus } R \text { is a } \gamma \text {-matrix. }
\end{aligned}
$$

Also $\quad \lim _{n \rightarrow \infty} \sum_{k=0}^{\infty} \boldsymbol{r}_{n, k+1} c_{k} \leftrightarrows \lim _{n \rightarrow \infty}\left\{\rho_{n} \sum_{k=0}^{\infty} \cdot r_{n, k} c_{k}\right\} \rightleftarrows \lim _{n \rightarrow \infty} \sum_{k=0}^{\infty} r_{n, k} c_{k}$.

Hence $R$ is regular. If $z_{0}$ is on the circle of convergence of $\Sigma a_{k} z^{k}$, representing $f(z)$,

$\sigma_{n} \equiv \sum_{k=0}^{\infty} r_{n, k} a_{k} z_{0}^{k}=\rho_{n} \sum_{k=0}^{\infty} a_{k}\left(\rho_{n} z_{0}\right)^{k} \quad$ converges to $\quad \rho_{n} f\left(\rho_{n} z_{0}\right)$.

Hence $\lim _{n \rightarrow \infty} \sigma_{n}=\lim _{\rho_{n} \rightarrow 1} f\left(\rho_{n} z_{0}\right)$ whenever the limit on the right-hand side exists. This proves the last statement. 


$$
\begin{array}{r}
\text { Examples. (a) If } \rho_{n}=\theta^{1 /(n+1)}, 0<\theta<1 \text {, then } r_{n, k}=\theta^{(k+1) /(n+1)} \\
\text { (b) If } \rho_{n}=(n+\beta)-p /(n+1), p>0, \beta>0, \\
\text { then } r_{n, k}=(n+\beta)^{-p(k+1) /(n+1) .}
\end{array}
$$

The matrix $R$ can be constructed independently of the series to which it applies. A somewhat similar construction can be used for a particular class of Dirichlet series, given in the usual notation as

(4.1) $\Sigma a_{k} \exp \{-\lambda(k) s\}$, representing the function $f(s)$ where $s=\sigma+i t$, where $\lambda(k) \rightarrow \infty$ with $k, \quad 0<\lambda(k)<\lambda(k+1)$.

Given the class of series characterized by $\{\lambda(k)\}$, we construct the matrix $L$ as follows:

We define a sequence $0<\mu(1)<\mu(2)<\ldots$ where $\mu(n) \rightarrow \infty$, and make

$$
l_{n, k}=\exp \{-p \lambda(k) / \mu(n)\}, \quad p>0, \quad n, k=1,2,3, \ldots
$$

[4.II] If the series (4.1) has a finite abscissa of convergence $s_{0}$, then the $\gamma$-matrix $L$ given by (4.2) sums the series at all points $s_{0}=\sigma_{11}+$ it of its. line of convergence at which $f\left(s_{0}+0\right)$ exists, and the sum is $f\left(s_{0}+0\right)$.

Proof: By (4.2) $l_{n, k}>l_{n, k+1}>0$ for every $n, k \geqq 1$,

and $\quad l_{n, k} \rightarrow 0$ for a fixed $n$ as $k \rightarrow \infty$,

$l_{n, k} \rightarrow 1$ for a fixed $k$ as $n \rightarrow \infty$.

Hence

$$
\sum_{k=1}^{\infty}\left|l_{n, k}-l_{n, k+1}\right|=\sum_{k=1}^{\infty}\left(l_{n, k}-l_{n, k+1}\right)=l_{n, 1} \leqq 1 .
$$

Thus $L$ is a $\gamma$-matrix. Also for $s_{0}=\sigma_{0}+i t$

$$
\begin{aligned}
S_{n} \equiv \sum_{k=1}^{\infty} l_{n, k} a_{k} \exp \left\{-\lambda(k) s_{0}\right\} & =\sum_{k=1}^{\infty} a_{k} \exp \left[-\lambda(k)\left\{s_{0}+p / \mu(n)\right\}\right] \\
& =f\left\{s_{0}+p / \mu(n)\right\},
\end{aligned}
$$

and therefore $S_{n} \rightarrow f\left(s_{0}+0\right)$ as $n \rightarrow \infty$ whenever the limit exists. This concludes the proof.

Example. For the class of special Dirichlet series $\Sigma a_{k} / k^{s}=\Sigma a_{k} \exp (-s \log k)$ the matrix $L$ is given by

$$
l_{n, k}=\exp \{-p \log k / \mu(n)\}=k^{-p / \mu(n)} .
$$

Birkbeck College, UNIVERSITY OF LONDON. 